\section{Rückkehr der sozialen Dimension}

\author{
Zum ersten Mal seit langem hat das Nobelpreiskomitee mit der Ehrung von \\ Amartya K. Sen die soziale Dimension des Wirtschaftens und die Bedeutung \\ von Fragen der Einkommensverteilung in Erinnerung gerufen. Mit seinen \\ Arbeiten hat Sen u.a. Grundlagen gelegt, an denen die akfuelle Diskussion \\ um soziale Nachhaltigkeit und ihre Kriterien nicht vorbeikommen wird.
}

A Von Volker Caspari martya Kumar Sen wurde 1933 in Santiniketan (Bengalen) in Indien geboren. Zunächst studierte er in Kalkutta und nach Abschluß des Baccalaureus dann ab 1954 Ökonomie in Cambridge/ Großbritannien, wo er 1959 promovierte. Nach Professuren in Kalkutta und Delhi, an der London School of Economics und in Oxford war Sen von 1989 bis 1997 Lament University Professor für Ökonomie und Philosophie an der Harvard University. Seit 1998 ist er als Master des Trinity College an seine Alma Mater nach Cambridge zurückgekehrt.

Sen hat sich kritisch und konstruktiv mit dem Utilitarismus auseinandergesetzt. In einem seiner zentralen Arbeitsgebiete - der Theorie kollektiver Entscheidungen - knüpfte er an Kenneth Arrows Unmöglichkeitstheorem an. Arrow wies nach, daß eine logisch konsistente Werteordnung der Gesellschaft als Derivat der individuellen Präferenzen ihrer Mitglieder als nicht realisierbar verworfen werden kann. Sen bewies darüber hinaus, daß das politische Prinzip der Liberalität, verstanden als die Bedingung minimaler individueller Rechte, und das Pareto-Prinzip bei der Aufstellung einer konsistenten gesellschaftlichen Werteordnung in einen Widerspruch geraten (die Unmöglichkeit des ,,paretianischen Liberalen“). Vor allem auf diesen Arbeiten beruht Sens Ruf in der ,scientific community“.

Sen hat in theoretischen und empirischen Arbeiten das Thema „Gerechtigkeit“ und damit das Verteilungsproblem, das Ökonomen seit dem Übergang zur ordinalen Nutzentheorie weitgehend mieden, in die Wohlfahrtsökonomik zurückgebracht.

Entwicklungsökonomen kennen A. Sen als den Autor von Poverty and Famines: An Essay on Entitlement and Deprivation (1981) und verorten ihn in der Armutsforschung. Tatsächlich bilden seine theoretischen Arbeiten und seine empirisch-angewandten Untersuchungen eine innere Einheit. Um nämlich verschiedene
Volkswirtschaften hinsichtlich ihrer Wohlfahrtsverteilung miteinander vergleichen zu können, oder um die zeitliche Veränderung der Wohlfahrtsverteilung in einer Volkswirtschaft beurteilen zu können, benötigt man Indizes, die solche Änderungen abbilden. Die Konstruktion solcher Indizes ist eine direkte Anwendung der Wohlfahrtsökonomik. So hat A. Sen vor allem wertvolle Beiträge zur Theorie der Armutsindizes beigesteuert, indem er u.a. gezeigt hat, daß das oft verwendete Maß ,Anteil der Gesamtbevölkerung unterhalb eines arbiträr festgelegten Mindesteinkommens" keine informative Maßzahl darstellt, weil nämlich deutliche Verbesserungen in der materiellen Versorgung dieses Bevölkerungsanteils diese Maßzahl solange nicht erhöht, wie das Mindesteinkommen nicht überschritten wird.

\section{- Wohlfahrt und Verteilung}

Auch hinsichtlich der Konstruktion von Wohlfahrtsindizes hat Sen darauf verwiesen, daß nicht allein das Realeinkommen, also die Menge der Güter über die eine Person verfügen kann, Wohlfahrt erzeugt, sondern auch, was eine Person mit diesen Gütern erreichen kann. Damit hängt die Wohlfahrt einer Person auch maßgeblich von der Ausstattung mit Befähigungen ab. Beispielsweise ermöglicht ein entsprechend hohes Realeinkommen einer Person den Kauf eines PCs. Das Spektrum seiner Nutzungsmöglichkeiten hängt freilich maßgeblich von den Kenntnissen der Person ab. Bei ,geringen“ Kenntnissen kann sie nur damit spielen, bei erweiterten Kenntnissen eröffnet sich ein ganzes Spektrum von Nutzungsmöglichkeiten. Da die Befähigungen von Personen maßgeblich von solchen Faktoren wie z.B. Gesundheit, Bildungschancen, Infrastruktur, Umwelt etc. beeinflußt werden, sind diese Faktoren in Wohlfahrtsindikatoren zu berücksichtigen. So ist z. B. der Human Development Index der UN ein Indikator, der solche Aspekte berïcksichtigt.
Wenn kulturelle, politische und gesellschaftliche Verhältnisse sich auf die Wohlfahrtsverteilung auswirken, dann liegt es auf der Hand, daß diese Verhältnisse auch bei besonders tragischen Formen der Wohlfahrtsverteilung, bei Hungersnöten nämlich, eine nicht zu unterschätzende Rolle spielen. Sen hat durch die Analyse einer ganzen Reihe großer Hungersnöte in Indien, Bangladesch sowie in der Sahel-Zone zeigen können, daß Hungersnöte nicht allein auf eine Nahrungsmittelknappheit zurückgeführt werden können. Wenn nämlich Hungersnöte auftreten, obwohl die Nahrungsmittelproduktion unverändert ist, muß die Ursache woanders gesucht werden. So zeigt er, daß eine wesentliche Ursache der Hungersnot im Jahr 1974 in Bangladesch auf den durch die Überflutung erzeugten Anstieg der Nahrungsmittelpreise zurückzuführen ist. Da durch die Überflutung die Felder nicht bebaut werden konnten, verloren die Landarbeiter ihre Haupteinkommensquelle. Die Folge war, daß in dieser gesellschaftlichen Schicht überproportional viele Menschen verhungerten. In Kenntnis dieser Mechanismen hat A. Sen Ideen und wirtschaftspolitische Instrumente vorgeschlagen, die erstens dazu beitragen können, Hungersnöte zu verhindern oder zweitens deren Ausmaß einzudämmen, wenn sie bereits ausgebrochen sind.

Mit der Verleihung des Nobelpreises 1998 für Wirtschaftswissenschaften an Amartya Sen hat die Königlich Schwedische Akademie der Wissenschaften einen Wissenschaftler geehrt, der im Zeitalter der Spezialisierung wieder näher aneinanderführte was einmal Eins war, nämlich Ökonomik und praktische Philosophie. In der aktuellen Diskussion um Nachhaltige Entwicklung ist dies aktueller denn je.

\section{Ausgewählte Literatur}

A. Sen: Collective Choice and Social Welfare, Elsevier Science 1997.

- A. Sen: Recources, Values and Development, Harvard University Press, 1984.

- A. Sen: On Ethics and Economics, Blackwell Publishers, Reprint 1989.

\section{Der Autor}

Dr. Volker Caspari ist Professor für Volkswirtschaftslehre an der TU Darmstadt.

Kontakt: Fachbereich 1, Residenzschloß, 64283 Darmsiadt, Tel. 06151/ 16-2119, Fax -5553,

E-mail: caspari@vwl.tu-darmstadt.de 
(c) 20I0 Authors; licensee IÖW and oekom verlag. This is an article distributed under the terms of the Creative Commons Attribution Non-Commercial No Derivates License (http://creativecommons.org/licenses/by-nc-nd/3.o/), which permits unrestricted use, distribution, and reproduction in any medium, provided the original work is properly cited. 